\title{
Static Optimization via Tracking of the Necessary Conditions of Optimality using Neighboring Extremals
}

\author{
S. Gros, B. Srinivasan, and D. Bonvin \\ Laboratoire d'Automatique \\ École Polytechnique Fédérale de Lausanne \\ CH-1015 Lausanne, Switzerland
}

\begin{abstract}
In the framework of process optimization, the use of measurements to compensate the effect of uncertainty has re-emerged as an active area of research. One of the ideas therein is to directly track the necessary conditions of optimality (NCO tracking). NCO tracking for steady-state optimization consists of monitoring the active constraints and pushing certain sensitivities to zero. In standard extremumseeking controllers, the sensitivities are evaluated by perturbation of the inputs, which can be experimentally expensive. Fortunately, more measurements (typically the outputs) than just the cost function are available. The idea is to incorporate the output measurements in the optimization framework for estimating the sensitivities, and thereby implementing the update laws. It is shown that this approach can bring the system fairly close to the optimum with relatively few experimental trials.

Keywords: Static optimization, Measurement-based optimization, Gradient estimation, NCO tracking, Neighboring extremals, Feedback control
\end{abstract}

\section{INTRODUCTION}

The optimization of dynamic systems amidst uncertainty has re-gained popularity recently [7], [15], [17], [16] after some initial work in the 1950s [9], [10], [11]. This is mainly due to advances in instrumentation and thus to the availability of more measurements. In this context, two optimization classes need to be distinguished: (i) optimization of a steady-state operating point [8], [7], and (ii) optimization of input profiles [16], [5]. The former can be treated as a static optimization problem (dynamic systems operated at steady state), while the latter requires dynamic optimization tools. It can be argued that the former has considerable industrial impact due to the large equipment size and production volume associated with these processes. Examples of continuous processes are numerous in the process industry, such as continuous chemical production or mineral processing.

When production is performed at steady state, it is critical that the system be operated as close to the optimum as possible. Standard optimization tools rely on a process model which, for industrial applications, are typically inaccurate or incomplete. Model mismatch is usually the result of simplifications and process variations [3], [1], [13]. Hence, the optimal inputs computed from the available models are often not optimal for the reality.

On the other hand, measurement-based optimization uses appropriate measurements to compensate the effect of uncertainty. The measurements are used to either: (i) adapt the parameters of a process model and re-optimize it (explicit optimization) [8], [17], [4], or (ii) directly adapt the inputs (implicit optimization) [7], [15], [16]. Furthermore, in static implicit optimization, it is important to distinguish between three types of techniques:

1) Zero-order methods - In techniques labeled evolutionary optimization [2], a simplex type algorithm is used to approach the optimum. For every combination of operating conditions, the cost function is measured experimentally.

2) Gradient-based methods - In techniques labeled extremum-seeking control [7], the gradients are estimated experimentally using a sinusoidal excitation. The excitation frequency needs to be sufficiently small for a time-scale separation between the system dynamics and the excitation frequency to exist. This scheme also uses only the measurement of the cost function.

3) Reformulation methods - In techniques labeled selfoptimizing control [15], the optimization problem is recast as a problem of choosing outputs whose optimal values are invariant to uncertainty. The output values as such vary with uncertainty but are brought back to their invariant set points using measurements. This scheme uses the output information rather than the measurement of the cost function.

This paper presents a gradient-based approach where first-order Taylor series approximations of the gradients are used. Moreover, it uses the entire set of measurements, i.e. output information. Hence, it can compute the gradients in one go, which cannot be done when only the cost function is measured. Thus, the number of iterations required to get to the optimum is considerably lower than with a perturbation (extremum-seeking) approach. The determination of the gradients is based on neighboring extremals that use the entire state information. Though measurements of all the states is rarely available, estimation techniques (software sensors) have reached a sufficient level of maturity to compute this information [12], [6], [14].

This paper is organized as follows. Section II formulates the optimization problem. Section III details the neighboring-extremal scheme and brings a proof of its local stability and the link to perturbation approaches. An isothermal continuous reactor example is considered in 
Section IV, while Section V provides the conclusions.

\section{PRELIMINARIES}

\section{A. Problem Formulation}

The standard steady-state optimization problem consists of minimizing a given cost function under equality and inequality constraints. At the optimum, some of the inequality constraints are active, and a standard assumption is that the set of active constraints does not change with uncertainty. In such a case, these active constraints can be kept active under uncertainty using simple controllers, which in turn removes certain degrees of freedom from the optimization problem. Thus, a problem without inequality constraints and a smaller set of decision variables can be formulated as is done in most approaches [15], [7], [17].

The general formulation of a static optimization problem without inequality constraints is considered:

$$
\begin{array}{ll} 
& \min _{u} \Phi(x, u, \theta) \\
\text { s.t. } & F(x, u, \theta)=0
\end{array}
$$

where $\Phi$ is the smooth scalar cost function to be minimized, $u$ the $m$-dimensional vector of inputs, $x$ the $n$-dimensional vector of states, $\theta$ the $n_{\theta}$-dimensional vector of parameters, and $F$ the $n$-dimensional vector of algebraic equations that describe the dynamic system at steady state. Note that the system equations $F$ can be solved for $x$ and substituted into $\Phi$. Such a simplification is not done here in purpose since the information on $x$ will be used explicitly to compute the sensitivities.

Introducing the Lagragian $L=\Phi+\lambda^{T} F$, where $\lambda$ are the adjoints of the system, the necessary conditions of optimality for (1)-(2) are:

$$
L_{u}=0, \quad L_{x}=0, \quad L_{\lambda}=F=0
$$

The notation $a_{b}=\frac{\partial a}{\partial b}$ will be used in this paper. Assuming that $F_{x}$ is invertible, the condition $L_{x}=0$ defines the adjoints:

$$
\lambda^{T}=-\Phi_{x} F_{x}^{-1}
$$

while the condition $L_{u}=0$ represents the condition of optimality. Using (4) in $L_{u}=0$ gives:

$$
L_{u}=\Phi_{u}-\Phi_{x} F_{x}^{-1} F_{u}=\left(\frac{\partial \Phi}{\partial u}\right)^{+}=0
$$

with $\left(\frac{\partial \Phi}{\partial u}\right)^{+}$being the total derivative that takes into account the direct effect of $u$ and the effect of $u$ through $x$.

\section{B. Gradient-based Approach to Measurement-based Opti-} mization

The goal of the gradient-based approach is to find an update $\delta u$ of the inputs that pushes the total derivative to zero. The first variation of the total derivative is given by:

$$
\delta\left(\frac{\partial \Phi}{\partial u}\right)^{+}=\left(\frac{\partial^{2} \Phi}{\partial u^{2}}\right)^{+} \delta u+\left(\frac{\partial^{2} \Phi}{\partial u \partial \theta}\right)^{+} \delta \theta
$$

Hence, for a variation $\delta \theta$ of the parameters, the corresponding $\delta u$ that forces the first variation of the sensitivity to zero is:

$$
\begin{aligned}
\delta u= & -\left[\left(\frac{\partial^{2} \Phi}{\partial u^{2}}\right)^{+}\right]^{-1}\left(\frac{\partial^{2} \Phi}{\partial u \partial \theta}\right)^{+} \delta \theta= \\
& -\left[\left(\frac{\partial^{2} \Phi}{\partial u^{2}}\right)^{+}\right]^{-1}\left(\frac{\partial \Phi}{\partial u}\right)_{\text {meas }}^{+}
\end{aligned}
$$

The term $\left(\frac{\partial^{2} \Phi}{\partial u \partial \theta}\right)^{+} \delta \theta$ describes, for a constant $u$, the variation of the total derivative due to the variation of $\theta$. In other words, if, for a constant $u$, the total derivative is estimated using a perturbation approach, the measured quantity $\left(\frac{\partial \Phi}{\partial u}\right)_{\text {meas }}^{+}$is, to a first-order approximation, $\left(\frac{\partial^{2} \Phi}{\partial u \partial \theta}\right)^{+} \delta \theta$.

\section{AdAptation using Neighboring-Extremal FEEDBACK}

\section{A. Formulation of the Update Law}

Neighboring-extremal controllers are based on the variations of the conditions of optimality. Also, they assume that the parameters do not change from one step to another (or at least the variation is insignificant). Given the measurements at time instant $k$, i.e. $\left(\delta u_{k}, \delta x_{k}\right)$, the variational equations (3) for the next step $k+1$ becomes:

$$
\begin{aligned}
L_{u x} \delta x_{k+1}+L_{u u} \delta u_{k+1} & +F_{u}^{T} \delta \lambda_{k+1} \\
& +L_{u \theta} \delta \theta=0 \\
L_{x x} \delta x_{k+1}+L_{x u} \delta u_{k+1} & +F_{x}^{T} \delta \lambda_{k+1} \\
& +L_{x \theta} \delta \theta=0 \\
F_{x} \delta x_{k+1}+F_{u} \delta u_{k+1} & +F_{\theta} \delta \theta=0
\end{aligned}
$$

In addition, the last equation can also be written for the step $k$ :

$$
F_{x} \delta x_{k}+F_{u} \delta u_{k}+F_{\theta} \delta \theta=0
$$

The 4 equations (8)-(11) contain the 4 unknowns $\delta x_{k+1}$, $\delta u_{k+1}, \delta \theta$ and $\delta \lambda_{k+1}$ and can be solved in terms of the current measurements $\left(\delta u_{k}, \delta x_{k}\right)$.

From (11), $\delta \theta=-F_{\theta}^{+}\left(F_{x} \delta x_{k}+F_{u} \delta u_{k}\right)$, where $F_{\theta}^{+}$is the Moore-Pendrose pseudo-inverse of $F_{\theta}$. Then, the optimal variations $\delta x_{k+1}, \delta u_{k+1}, \delta \lambda_{k+1}$ are obtained by solving the system of equations (8)-(10):

$$
\begin{aligned}
{\left[\begin{array}{l}
\delta x_{k+1} \\
\delta u_{k+1} \\
\delta \lambda_{k+1}
\end{array}\right]=} & {\left[\begin{array}{ccc}
L_{u x} & L_{u u} & F_{u}^{T} \\
L_{x x} & L_{x u} & F_{x}^{T} \\
F_{x} & F_{u} & 0
\end{array}\right]^{-1} } \\
& {\left[\begin{array}{c}
L_{u \theta} \\
L_{x \theta} \\
F_{\theta}
\end{array}\right] F_{\theta}^{+}\left(F_{x} \delta x_{k}+F_{u} \delta u_{k}\right) }
\end{aligned}
$$


The solution for $\delta u_{k+1}$ gives:

$$
\begin{aligned}
\delta u_{k+1}= & K \delta \theta=-K_{x} \delta x_{k}-K_{u} \delta u_{k} \\
K= & -\left(L_{u u}-L_{u x} F_{x}^{-1} F_{u}-F_{u}^{T} F_{x}^{-T} L_{x u}\right. \\
& \left.+F_{u}^{T} F_{x}^{-T} L_{x x} F_{x}^{-1} F_{u}\right)^{-1} \\
& \left(L_{u \theta}-L_{u x} F_{x}^{-1} F_{\theta}-F_{u}^{T} F_{x}^{-T} L_{x \theta}\right. \\
& \left.+F_{u}^{T} F_{x}^{-T} L_{x x} F_{x}^{-1} F_{\theta}\right) \\
K_{x}= & K F_{\theta}^{+} F_{x} \\
K_{u}= & K F_{\theta}^{+} F_{u}
\end{aligned}
$$

\section{B. Convergence Analysis of the Proposed Adaptation Scheme}

It will be shown next that, in an ideal scenario, the proposed update converges in at most two steps. First a supporting lemma is established.

Lemma 1: For two matrix $A$ and $B, A$ being $m \times n$ and $B$ being $n \times m$, if $B A=0$, then the eigenvalues of $C=A B$ are all zeros.

Proof: $C=A B, C^{2}=A(B A) B=0$. Thus, $\mathrm{C}$ is nilpotent. If $C$ has an eigenvalue $\lambda \neq 0$, then there exists a vector $v$ such that $C v=\lambda v$, and $C^{2} v=\lambda^{2} v \neq 0$. This contradicts the fact that $C^{2}=0$. Hence, all eigenvalues of $C$ are zeros.

Theorem 1: The update law (13) converges locally to the optimum in at most two iterations.

\section{Proof:}

With the proposed update law (13), the variation $\delta x_{k+1}$ can be computed as:

$$
\begin{aligned}
\delta x_{k+1}= & -F_{x}^{-1} F_{\theta} \delta \theta-F_{x}^{-1} F_{u} \delta u_{k+1} \\
= & -F_{x}^{-1} F_{\theta} \delta \theta+F_{x}^{-1} F_{u} K F_{\theta}^{+} F_{u} \delta u_{k} \\
& +F_{x}^{-1} F_{u} K F_{\theta}^{+} F_{x} \delta x_{k}
\end{aligned}
$$

Hence, the evolution of $\delta x$ and $\delta u$ can can be written as the following discrete-time system:

$$
\left[\begin{array}{l}
\delta x_{k+1} \\
\delta u_{k+1}
\end{array}\right]=M\left[\begin{array}{l}
\delta x_{k} \\
\delta u_{k}
\end{array}\right]-\left[\begin{array}{c}
F_{x}^{-1} F_{\theta} \\
0
\end{array}\right] \delta \theta
$$

with

$$
M=\left[\begin{array}{cc}
F_{x}^{-1} F_{u} K F_{\theta}^{+} F_{x} & F_{x}^{-1} F_{u} K F_{\theta}^{+} F_{u} \\
-K F_{\theta}^{+} F_{x} & -K F_{\theta}^{+} F_{u}
\end{array}\right]
$$

Note that $M$ can be written as $M=A B$ with

$$
\begin{aligned}
A & =\left[\begin{array}{c}
F_{x}^{-1} F_{u} \\
-I
\end{array}\right] \\
B & =K F_{\theta}^{+}\left[\begin{array}{ll}
F_{x} & F_{u}
\end{array}\right]
\end{aligned}
$$

Also note that $B A=0$. Thus, applying Lemma 1, it can be seen that all eigenvalues are zero. This corresponds to a discrete plant with a dead-beat controller. Since $M^{2}=0$, it takes at most two iterations for convergence. The iterations converge to

$$
\left[\begin{array}{l}
\delta x^{*} \\
\delta u^{*}
\end{array}\right]=-(I-M)^{-1}\left[\begin{array}{c}
F_{x}^{-1} F_{\theta} \\
0
\end{array}\right] \delta \theta
$$

If $F_{\theta}^{+} F_{\theta}=I$, then it can be verified that

$$
(I-M)\left[\begin{array}{c}
-F_{x}^{-1}\left(F_{u} K-F_{\theta}\right) \\
K
\end{array}\right]=\left[\begin{array}{c}
-F_{x}^{-1} F_{\theta} \\
0
\end{array}\right]
$$

which leads to

$$
\left[\begin{array}{l}
\delta x^{*} \\
\delta u^{*}
\end{array}\right]=\left[\begin{array}{c}
-F_{x}^{-1}\left(F_{u} K-F_{\theta}\right) \\
K
\end{array}\right] \delta \theta
$$

which is in fact the solution of (8)-(10) for a given $\delta \theta$.

\section{Approximation of the Total Derivative}

The neighboring-extremal scheme provides a feedback adaptation law computed from the linearization of the system around the nominal operating point. In this section, it will be shown that the computation of the gradients from the state measurements is implicit in the feedback law. In particular, it will be shown that the update law (13) is equivalent to (7) with $\left[\left(\frac{\partial^{2} \Phi}{\partial u^{2}}\right)^{+}\right]^{-1}$ being computed from the nominal model and $\left(\frac{\partial \Phi}{\partial u}\right)_{\text {meas }}^{+}$reconstructed from the state and input measurements.

Proposition 1: The update law (13) is equivalent to (7) with

$$
\begin{aligned}
\left(\frac{\partial^{2} \Phi}{\partial u^{2}}\right)^{+}= & \left(L_{u u}-L_{u x} F_{x}^{-1} F_{u}-F_{u}^{T} F_{x}^{-T} L_{x u}\right. \\
& \left.+F_{u}^{T} F_{x}^{-T} L_{x x} F_{x}^{-1} F_{u}\right) \\
\left(\frac{\partial \Phi}{\partial u}\right)_{\text {meas }}^{+}= & \left(L_{u \theta}-L_{u x} F_{x}^{-1} F_{\theta}-F_{u}^{T} F_{x}^{-T} L_{x \theta}\right. \\
& \left.+F_{u}^{T} F_{x}^{-T} L_{x x} F_{x}^{-1} F_{\theta}\right) \\
& F_{\theta}^{+}\left(F_{x} \delta x_{k}+F_{u} \delta u_{k}\right)
\end{aligned}
$$

Proof:

Noting that from (5) $\left(\frac{\partial \Phi}{\partial u}\right)^{+}=L_{u}$, the variation of the total derivative is given by:

$$
\delta L_{u}=L_{u x} \delta x+L_{u u} \delta u+F_{u}^{T} \delta \lambda+L_{u \theta} \delta \theta
$$

Since the system equations are satisfied also in the presence of uncertainty, one obtains from:

$$
\delta L_{\lambda}=\delta F=F_{x} \delta x+F_{u} \delta u+F_{\theta} \delta \theta=0
$$

the corresponding variation of the state $x$ :

$$
\delta x=-F_{x}^{-1} F_{u} \delta u-F_{x}^{-1} F_{\theta} \delta \theta
$$

Also, the variation of $L_{x}$

$$
\begin{aligned}
\delta L_{x} & =L_{x x} \delta x+L_{x u} \delta u+F_{x}^{T} \delta \lambda+L_{x \theta} \delta \theta \\
& =0
\end{aligned}
$$

can be used to evaluate the variation of the Lagrange multipliers $\lambda$. Combining (29) and (30), one obtains:

$$
\begin{aligned}
\delta \lambda= & F_{x}^{-T} L_{x x} F_{x}^{-1} F_{u} \delta u+F_{x}^{-T} L_{x x} F_{x}^{-1} F_{\theta} \delta \theta \\
& -F_{x}^{-T} L_{x u} \delta u-F_{x}^{-T} L_{x \theta} \delta \theta
\end{aligned}
$$


Using (29) and (31) in (27) gives:

$$
\begin{aligned}
\delta L_{u}= & \left(L_{u u}-L_{u x} F_{x}^{-1} F_{u}-F_{u}^{T} F_{x}^{-T} L_{x u}\right. \\
& \left.+F_{u}^{T} F_{x}^{-T} L_{x x} F_{x}^{-1} F_{u}\right) \delta u \\
& +\left(L_{u \theta}-L_{u x} F_{x}^{-1} F_{\theta}-F_{u}^{T} F_{x}^{-T} L_{x \theta}\right. \\
& \left.+F_{u}^{T} F_{x}^{-T} L_{x x} F_{x}^{-1} F_{\theta}\right) \delta \theta
\end{aligned}
$$

Forcing $\delta L_{u}$ to zero provides the update law (13), where the evaluation of $\delta \theta$ is from the previous measurements, $\delta \theta=F_{\theta}^{+}\left(F_{x} \delta x_{k}+F_{u} \delta u_{k}\right)$. Comparing (32) with (6) gives (25) and (26).

The update law (13) establishes the neighboring-extremal approach as a means of obtaining a first-order approximation of the total derivative using auxiliary measurements. The gradient computation is in one go, as opposed to the case where multiple iterations are typically needed to evaluate the gradient with only the cost function being measured. Thus, the number of iterations required to get to the optimum is considerably reduced by using more information than just the cost function.

The neighboring-extremal approach uses the entire state information; however, measurements of all the states is rarely available. Yet, estimation techniques (software sensors) have reached a sufficient level of maturity to compute this information [12], [6], [14]. Combined with state estimation, the neighboring-extremal approach provides a reasonable alternative to experimental gradient evaluation.

\section{EXAMPLE}

\section{A. System Description}

An isothermal continuous chemical reactor with the two reactions $A+B \rightarrow C$ and $2 B \rightarrow D$ is considered. There are two feeds with the flow rates $F_{A}$ and $F_{B}$ of concentrations $c_{A_{i n}}$ and $c_{B_{i n}}$, respectively. The objective is to maximize the amount of $C$ produced, $\left(F_{A}+F_{B}\right) c_{C}$ while also taking into consideration the selectivity factor $\frac{\left(F_{A}+F_{B}\right) c_{C}}{F_{A} c_{A_{i n}}}$ as well as the weighted norm of the input flow rates $\frac{1}{2}\left[\begin{array}{ll}F_{A} & F_{B}\end{array}\right] R\left[\begin{array}{ll}F_{A} & F_{B}\end{array}\right]^{T}$. The optimization problem can be formulated mathematically as:

$$
\begin{aligned}
\max _{F_{A}, F_{B}} J= & \left(F_{A}+F_{B}\right) c_{C} \frac{\left(F_{A}+F_{B}\right) c_{C}}{F_{A} c_{A_{i n}}} \\
\text { s.t. } \quad & \frac{1}{2}\left[\begin{array}{ll}
F_{A} & F_{B}
\end{array}\right] R\left[\begin{array}{l}
F_{A} \\
F_{B}
\end{array}\right] \\
& F_{A} c_{A_{i n}}-\left(F_{A}+F_{B}\right) c_{A}-k_{1} c_{A} c_{B} V \\
& =0 \\
& F_{B} c_{B}-\left(F_{A}+F_{B}\right) c_{B}-k_{1} c_{A} c_{B} V \\
& -2 k_{2} c_{B}^{2} V=0 \\
& -\left(F_{A}+F_{B}\right) c_{C}+k_{1} c_{A} c_{B} V=0
\end{aligned}
$$

where $c_{X}$ is the concentration of species $X, k_{1}, k_{2}$ the rate constants. Model parameters and operating conditions are given in Table I.

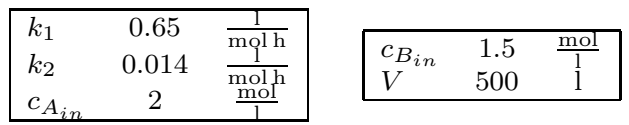

TABLE I

MODEL PARAMETERS AND OPERATING CONDITIONS

The parameter $k_{1}$ is considered uncertain between 0.3 and $3 \frac{1}{\mathrm{molh}}$. The input weight matrix $R$ is chosen as the identity matrix $I_{2 \times 2}$.

\section{B. Neighboring-extremal Approach}

The feedback gains obtained for the nominal point corresponding to $k_{1}=0.65$ are:

$$
\begin{aligned}
K_{x} & =\left[\begin{array}{lll}
1.2657 & 1.3613 & -0.0304 \\
1.6116 & 1.7332 & -0.0387
\end{array}\right] \\
K_{u} & =\left[\begin{array}{ll}
-0.0614 & -0.0499 \\
-0.0781 & -0.0635
\end{array}\right]
\end{aligned}
$$

Figure 1 compares, for various values of $k_{1}$, the optimal operating points obtained with the NE approach after 3 evaluations with the true optimal solution. The NE approach finds the optimal solution for all values of $k_{1}$, though the controller has been computed for $k_{1}=0.65$. Note that the NE controller does not know the value of $k_{1}$ used in the simulation and reaches the optimum without this knowledge.
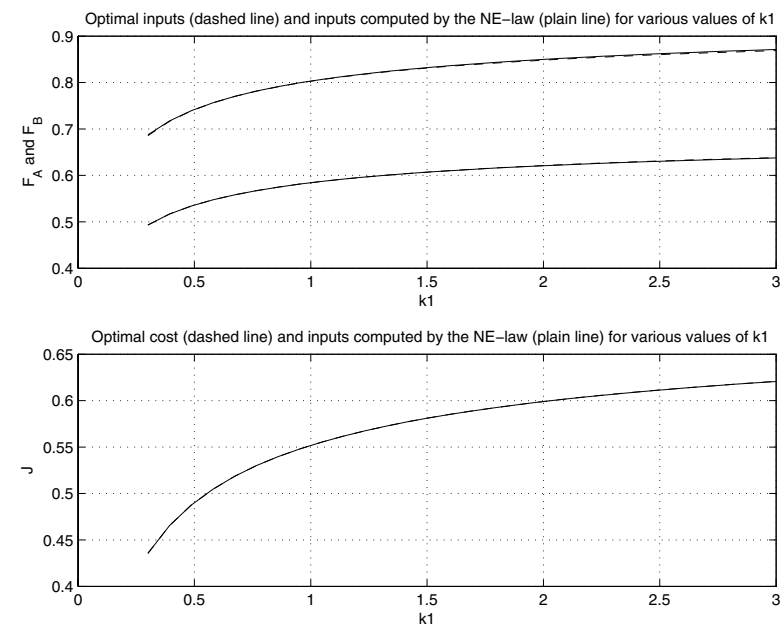

Fig. 1. Performance of the neighboring-extremal approach (after 3 evaluations) for various values of $k_{1}$ (true optimum in dashed line, NE in solid line)

Next, the simulation results presented in Figure 2 for $k_{1}=3$ compare the performance of the classical numericalperturbation approach with the NE-update approach. The main advantage of the NE controller over a perturbation approach is its fast convergence. While the NE controller converges within a few iterations using very simple computations, other methods often require a large number of 


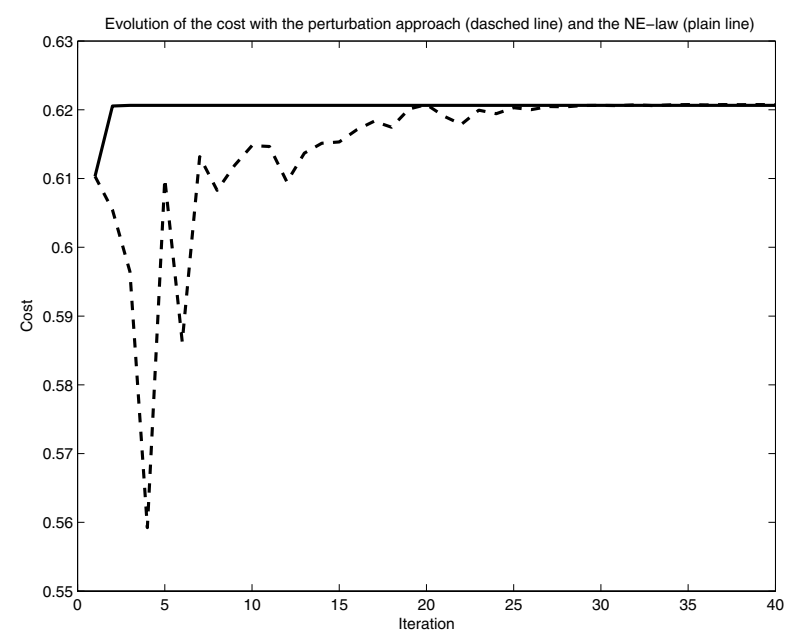

Fig. 2. Evolution of the cost using the neighboring-extremal approach and the perturbation approach for $k_{1}=3$

iterations and use more complex computations. In this simulation, the NE-based approach required 3 evaluations for the system to converge to its optimum, while the perturbation approach required between 42 and 63 evaluations for convergence depending on the value of $k_{1}$.

\section{CONCLUSION}

The measurement-based optimization of steady-state operating points has been addressed. A neighboring-extremal approach is used for computing the gradients as an alternative to the classical perturbation approach. The neighboringextremal approach uses the entire state information and requires far fewer iterations to get to the optimum because the sensitivity is implicitly computed in the feedback law rather than explicitly through experiments. The symbolic and numerical computations required to compute the feedback are rather straightforward and can be done off-line.

The main drawbacks of the neighboring-extremal approach is that the entire state information is required, which is often unavailable. Hence, state estimation is needed. Also, the proposed approach does not necessarily converge to the true optimum in the presence of uncertainty. The proof of convergence has been provided only for the nominal case. This sub-optimality introduced may be unacceptable in certain scenarios.

\section{REFERENCES}

[1] O. Abel and W. Marquardt. A model predictive control scheme for safe and optimal operation of exothermic semi-batch reactors. In IFAC DYCOPS-5, pages 761-766, Corfu, Greece, 1998.

[2] G. E. P. Box and N. R. Draper. Empirical Model-building and Response Surfaces. John Wiley, New York, 1987.

[3] J. W. Eaton and J. B. Rawlings. Feedback control of nonlinear processes using on-line optimization techniques. Comp. Chem. Eng., 14:469-479, 1990.

[4] J. Kadam and W. Marquardt. Towards integrated dynamic real-time optimization and control of industrial processes. In FOCAPO 2003 Fourth International Conference on Foundations of Computer-Aided Process Operations, Coral Springs, Florida, 2003.
[5] J. Kadam and W. Marquardt. Sensitivity-based solution updates in closed-loop dynamic optimization. In IFAC DYCOPS-7, Boston, Massachusetts, 2004.

[6] A. J. Koshkouei and A. S. I. Zinober. Sliding mode state observation for non-linear systems. International Journal of Control, 77(2):118127, 2004.

[7] M. Kristic and H-H. Wang. Stability of extremum seeking feedback for general nonlinear dynamic systems. Automatica, 36:595-601, 2000

[8] T. Marlin and A. N. Hrymak. Real-time operations optimization of continuous processes. In Chemical Process Control-V Conference, Tahoe City, Nevada, 1996.

[9] I. I. Morosanov. Method of extremum control. Automatic and Remote Control, 18:1077-1092, 1957.

[10] I. I. Ostrovskii. Extremum regulation. Automatic and Remote Control, 18:900-907, 1957.

[11] A. A. Pervozvanskii. Continuous extremum control system in the presence of random noise. Automatic and Remote Control, 21:673677, 1960.

[12] T. Rassi, N. Ramdani, and Y. Candau. Bounded error moving horizon state estimators for non-linear continuous-time systems:application to a bioprocess system. Journal of process control, 15(5):537-545, 2005.

[13] D. Ruppen, C. Benthack, and D. Bonvin. Optimization of batch reactor operation under parametric uncertainty - Computational aspects. J. Process Contr., 5(4):235-240, 1995.

[14] B. Sinopoli and L. Schenato. Kalman filtering with intermittent observations. IEEE Transactions on Automatic Control, 49(9):14531464, 2004

[15] S. Skogestad. Plantwide control: The search for the self-optimizing control structure. J. Process Contr., 10:487-507, 2000.

[16] B. Srinivasan, D. Bonvin, E. Visser, and S. Palanki. Dynamic optimization of batch processes: II. Role of measurements in handling uncertainty. Comp. Chem. Eng., 44:27-44, 2003.

[17] T. Zang, M. Guay, and D. Dochain. Adaptive extremum seeking control of continuous stirred tank bioreactors. AIChE J., 40(2):1020, 2001. 